Published in final edited form as:

Schizophr Res. 2020 February ; 216: 168-174. doi:10.1016/j.schres.2019.12.006.

\title{
Independent Support for Corticopallidal Contributions to Schizophrenia-Related Functional Impairment
}

\author{
Goda Tarcijonas ${ }^{1}$, William Foran ${ }^{1}$, Annie Blazer ${ }^{1}$, Shaun M. Eack ${ }^{1,2}$, Beatriz Luna ${ }^{1,3,4}$, \\ Deepak K. Sarpal ${ }^{1}$ \\ ${ }^{1}$ Department of Psychiatry, University of Pittsburgh, Pittsburgh, PA, USA \\ ${ }^{2}$ School of Social Work, University of Pittsburgh, Pittsburgh, PA, USA \\ ${ }^{3}$ Department of Psychology, University of Pittsburgh, Pittsburgh, PA, USA \\ ${ }^{4}$ Department of Pediatrics, University of Pittsburgh, Pittsburgh, PA, USA
}

\section{Abstract}

Background: Abnormalities between the prefrontal cortex and basal ganglia have been described by numerous studies of schizophrenia (SZ). We recently reported that individuals with first episode SZ who develop greater vocational and social impairments show lower baseline functional connectivity between the globus pallidus (GP) and regions of the intrinsic salience network. Here we extend these findings to probe the integrity of this system in individuals with chronic illness.

Methods: All data were obtained from a publicly available Center of Biomedical Research Excellence dataset (http://fcon_1000.projects.nitric.org/indi/retro/cobre.html) that included resting-state fMRI and structural scans, and an array of clinical and neuropsychological measures. Participants with SZ were divided into high- or low-functioning groups based on scores across measures of psychopathology and cognitive functioning. Corticopallidal functional connectivity was examined between low- and high-functioning individuals with SZ and matched healthy control participants. We focused on connectivity between GP structures and a priori regions of the salience network that were significant in our previous study. Exploratory voxel-wise analyses were also conducted.

Results: Lower functioning individuals with SZ demonstrated less connectivity between bilateral GP externa and nodes within the salience network, relative to healthy controls. No connectivity differences were observed between low- and high-functioning individuals with SZ. Exploratory

\footnotetext{
Correspondence: Deepak K. Sarpal, M.D., 3501 Forbes Ave, Suite 530, Pittsburgh, PA 15213, sarpaldk@upmc.edu. Contributors

Shaun M. Eack, Deepak K. Sarpal and Beatriz Luna provided conceptual guidance for the project, while Deepak K. Sarpal and Goda Tarcijonas designed the analytic protocol and managed analyses. Goda Tarcijonas, William Foran, and Deepak K. Sarpal undertook the statistical analysis with guidance and support from Beatriz Luna. Deepak K. Sarpal, Shaun M. Eack, Goda Tarcijonas, and Annie Blazer shared duties in preparation of the manuscript. All authors contributed to and have approved the final manuscript.

Publisher's Disclaimer: This is a PDF file of an unedited manuscript that has been accepted for publication. As a service to our customers we are providing this early version of the manuscript. The manuscript will undergo copyediting, typesetting, and review of the resulting proof before it is published in its final form. Please note that during the production process errors may be discovered which could affect the content, and all legal disclaimers that apply to the journal pertain.
} 
voxel-wise analyses highlighted additional large-scale corticopallidal abnormalities in lowerfunctioning participants with SZ.

Conclusions: These results confirm our previous work in a more chronic cohort of individuals with SZ. Our findings further advance corticopallidal connectivity as a biomarker of functional impairments in SZ and lay the groundwork for treatment-based studies.

\section{Keywords}

globus pallidus; schizophrenia; functional connectivity; functional impairment; fmri; salience network

\section{INTRODUCTION}

Functional impairments are prominent features of schizophrenia (SZ) that significantly contribute to disability throughout the world (Bowie et al., 2006; Global Burden of Disease Study, 2015; Green, 1996; Jaaskelainen et al., 2013). It is estimated that less than $15 \%$ of individuals with SZ achieve a level of recovery consistent with normal functioning (Robinson et al., 2004). Broadly, functional impairment in schizophrenia manifests in multiple areas of everyday life, including the ability to maintain social relationships, sustain employment, and live independently. Both negative symptoms and neurocognitive deficits are established primary factors that influence the severity of functional impairment in individuals with SZ (Bowie et al., 2006; Fett et al., 2011; Green, 1996; Reddy et al., 2016). Though SZ-related functional impairment is well-characterized, robust, and consistent across cohorts and studies, therapeutic strategies are limited. All clinically available somatic treatments target only the positive symptoms of SZ with limited effect on long-term chronic disability. This lack of therapeutic approaches is partly due to a deficient understanding of neurobiological mechanisms related to functional impairment. Thus, elucidating neural contributions remains paramount for novel treatment development.

Accumulating evidence implicates large-scale cortico-basal ganglia-thalamo-cortical (CBGTC) circuitry in the neuropathology of SZ (Avram et al., 2018; Giraldo-Chica and Woodward, 2017; Meyer-Lindenberg et al., 2002). A subset of studies has also focused on CBGTC links in relation to clinical outcomes. Response to antipsychotic treatment has been linked to increased corticostriatal connectivity (Hadley et al., 2014; Sarpal et al., 2016; Sarpal et al., 2015). Elsewhere within CBGTC circuitry, links between the basal ganglia and prefrontal cortex have been implicated in overall functional outcomes of SZ (Wojtalik et al., 2017). We recently reported that functional connectivity between regions of the globus pallidus (GP) and the nodes within the salience network is decreased in individuals with first-episode schizophrenia who developed more severe functional impairment (Tarcijonas et al., 2019). This finding was independent of striatal connectivity, consistent with circuitry observed in akinetic mutism (Darby et al., 2018), and involved corticopallidal nodes that are targeted by neuromodulatory approaches such as deep brain stimulation (Da Cunha et al., 2015; Parvizi et al., 2013). Though encouraging, independent confirmation of this result is necessary for further biomarker development of this functional circuit. 
Given that our previous study focused on first-episode schizophrenia, it remains unknown whether GP-salience network connectivity related to global functional impairment in individuals with more chronic SZ. To address this question, we performed the present study using a publicly available dataset that included neuroimaging and clinical data from cohorts of individuals with chronic schizophrenia and matched healthy control participants. Our primary goal was to extend our initial analysis to an independent dataset of patients with chronic illness. No direct measure of global functioning was included in this dataset. However, to leverage available clinical and neurocognitive measures, we used a data-driven approach to derive novel proxy measures of global functioning, based on factors across clinical, social cognitive, and neurocognitive assessments. We tested the hypothesis that functional connectivity between GP structures and regions of the salience network, including and the insula and dorsal anterior cingulate cortex, significantly differ between lowfunctioning individuals with SZ and matched healthy controls (HC). Secondly, we were interested in whether corticopallidal findings exist in areas outside of the salience network, possibly implicating novel circuitry related to illness progression in this cohort of chronic participants with SZ. Thus, we also conducted exploratory whole-brain analyses.

\section{METHODS}

\subsection{Participants and Clinical Phenotyping}

We included participants from the publicly available Center of Biomedical Research Excellence (COBRE) dataset (Calhoun et al., 2011). The full dataset downloaded included 72 deidentified participants, aged 18-65, with a diagnosis of SZ, confirmed by a Structured Clinical Interview for DSM disorders by trained clinicians. A cohort of 74 matched and deidentified HCs was also downloaded. However, data from one HC participant did not properly pass our preprocessing methods (see below) due to incomplete volumes of data. As a conservative measure we left this participant out, leaving $73 \mathrm{HC}$ participants included in this study. More information about the dataset is available at http:// fcon_1000.projects.nitric.org/indi/retro/cobre.html. Exclusion criteria consisted of a history of substance abuse or dependence in the year prior to the assessment; a history of neurological disorders; intellectually disabled; and severe head trauma with loss of consciousness for more than $5 \mathrm{~min}$. Written informed consent was obtained from all subjects in accordance with the institutional review board of the University of New Mexico. The COBRE dataset is publicly distributed with a Creative Commons License.

All SZ participants underwent assessments for psychopathology with the Clinical Global Impressions scale (CGI) (Guy and Bonato), and Positive and Negative Syndrome Scale (PANSS) (Kay et al., 1987). Neurocognition was assessed with the Wechsler Adult Intelligence Scale (WAIS) (Wechsler, 2008), Wechsler Abbreviated Scale of Intelligence (WASI) (Wechsler, 1999), Measurement and Treatment Research to Improve Cognition in Schizophrenia Consensus Cognitive Battery (MCCB) (Nuechterlein et al., 2008), and the Mayer-Salovey-Caruso Emotional Intelligence Test (MSCEIT) (Mayer et al., 2003).

A subset of 61 SZ participants had usable data for the CGI, PANSS, WAIS, WASI, MCCB, and the MSCEIT assessments and were included in our analyses relating functional impairment with GP connectivity. Since scores on these assessments putatively predict 
functional outcomes in SZ, we were interested in identifying data-driven factors that explain the variance across them, each factor representing a unique contribution to functional impairment. Given the lack of a direct measure of functional impairment, this method, allowed for us to approximate independent representations of functional impairment with the cross-sectional set of clinical and neuropsychological batteries included in the COBRE dataset. To reduce our data, a principal component analysis was performed on scores or ratings across our assessments of interest. Horn's parallel analysis was then used to empirically identify the appropriate number of factors to retain in further analyses. Results of our analysis are represented in Table 1 and Supplementary Figure 1. Three principal components (PC) passed Horn's analysis and explained 37.2\% (PC1), 19.3\% (PC2), and $17.9 \%$ (PC3) of the variance across the clinical and neurocognitive assessments listed above. Each of these PCs represented an orthogonal contribution to overall functioning and were treated independently to characterize functional impairment in separate sets of neuroimaging analyses, as described in section 2.4 below. For each PC, low- and high-functioning patients with SZ were defined by a median split of the average loading of SZ participants' data onto it. This resulted in three PCs with different combinations of high and low-functioning SZ participants. A binary sub-categorization of SZ participants into low- and high-functioning groups mirrors our previous longitudinal study (Tarcijonas et al., 2019), and facilitates comparisons of neuroimaging data with HCs who did not undergo all clinical and neurocognitive assessments of interest. To our knowledge, this approach for categorizing SZ participants based on functioning has not been used in prior studies but allows for the utilization of cross-sectional data to examine functional impairment in the absence of a direct measure, such as the Strauss Carpenter Outcome Scale (Strauss and Carpenter, 1972).

\subsection{Neuroimaging acquisition}

Structural scans were acquired with a multi-echo MPRAGE (MEMPR) sequence with the following parameters: TR/TE/TI $=2530 /[1.64,3.5,5.36,7.22,9.08] / 900 \mathrm{~ms}$, flip angle $=7^{\circ}$, FOV $=256 \times 256 \mathrm{~mm}$, Slab thickness $=176 \mathrm{~mm}$, Matrix $=256 \times 256 \times 176$, Voxel size $=1 \times 1 \times 1$ $\mathrm{mm}$, Number of echos $=5$, Pixel bandwidth $=650 \mathrm{~Hz}$, Total scan time $=6 \mathrm{~min}$. Resting-state scans were acquired with single-shot full k-space echo-planar imaging (EPI) with ramp sampling correction using the inter-comissural line (AC-PC) as a reference (TR: 2 s, TE: 29 ms, matrix size: $64 \times 64,32$ slices, voxel size: $3 \times 3 \times 4 \mathrm{~mm}$ ).

\subsection{Neuroimaging Preprocessing}

Consistent with our previous work, preprocessing was completed in accordance with an established pipeline that uses tools from AFNI (https://afni.nimh/nih.gov/) and FSL (http:// www.fmrib.ox.ac.uk ) packages (Cox, 1996; Jenkinson et al., 2012). This pipeline has consistently minimized head motion-dependent confounds across multiple studies, including our previous work (Hallquist et al., 2013; Tarcijonas et al., 2019). Preprocessing steps included the following: 4D slice timing and motion correction with NIPy (http://nipy.org/); skull stripping; co-registration and warping to standard MNI space; spatial smoothing using a $5 \mathrm{~mm}$ full width at half maximum (FWHM) Gaussian kernel; high pass filtering at 100 volumes, and grand mean intensity normalization (10000/global median). To minimize noise in our data and account for head motion, including movement-related 'spikes', we used wavelet de-spiking (Patel et al., 2014), which uses a data-driven method to identify and 
remove movement-related artifacts. Following wavelet de-spiking, the following steps occurred: signal nuisance regression with motion, cerebrospinal fluid (CSF), white matter, and both CSF and white matter derivatives, and bandpass filtering between 0.009 and .08 $\mathrm{Hz}$.

\subsection{Functional Connectivity Analyses}

Due to differences in the resolution of scans in this study and our previous work, resampled regions of interest (ROIs) from our previous work resulted in seed ROIs that were 1 voxel in size given differences in resolution of scans from the two studies. To account for more variance in whole brain GP connectivity, we used anatomical masks of GP structures as seed ROIs. This resulted in 4 larger ROIs: left GP externa (24 voxels), left GP interna (8 voxels), right GP interna (11 voxels), right GP externa (26 voxels). Images of these ROIs are displayed in Figure 1. The mean time courses were extracted from all four GP ROIs and corresponding z-transformed whole-brain functional connectivity maps were generated for all $\mathrm{HC}$ and SZ participants with the extracted waveforms as a reference. For our primary analysis, which was interested in reproducing results from our previous findings, we focused on functional connectivity between our GP ROIs and peak nodes (with a $5 \mathrm{~mm}$ radius) from our previous work in the dorsal anterior cingulate $(x=1, y=13, z=42)$, left anterior insula $(\mathrm{x}=-33, \mathrm{y}=8, \mathrm{z}=8)$, and right anterior insula $(\mathrm{x}=40, \mathrm{y}=27, \mathrm{z}=3)$, all regions within the salience network (Figure 2). Our three ROIs were combined to create one tripart ROI that represented the salience network. We confirmed that all three of these nodes were inclusive within the salience network via an external meta-analytic search engine. We entered the term 'salience' into the neurosynth database (https://www.neurosynth.org) to generate a reverse inference map representing boundaries of meta-analytic activation of the salience network. We then visually confirmed that nodes of our mask were well within the resulting network (Yarkoni et al., 2011).

All results of our functional connectivity analyses were deemed significant if they surpassed Bonferroni correction ( $\mathrm{p}$ < 0.004: 0.05 divided by 12, which accounts for 3 PCs and 4 ROIs) (Gorgolewski et al., 2011). In post-hoc analyses functional connectivity results were examined along with age, and antipsychotic medication exposure in chlorpromazine equivalents. Finally, connectivity results were examined along with framewise displacement (FD), to confirm that our results were not influenced by head motion.

\subsection{Exploratory Group Analyses}

Whole-brain connectivity differences between each combination of pairs from our three participant groups (low-functioning SZ, high-functioning SZ, and HC) were examined for all four GP ROIs using 3dttest++ in AFNI. These analyses were repeated for each PC, which represented a unique factor related to functioning. Significance was defined voxel-wise at $\mathrm{p}<$ 0.001 , and with cluster correction at $\mathrm{p}<0.05$ with via $3 \mathrm{dClustSim}$ in AFNI. Options entered into $3 \mathrm{dClustSim}$ included values of the amount of smoothing present estimated by a spatial autocorrelation function, and 10,000 iterations. The corrected cluster threshold was defined at 59 contiguous voxels. 


\section{RESULTS}

\subsection{Demographics and Clinical Data}

Demographic and clinical data are displayed in Table 2. Data from $72 \mathrm{SZ}$ and $73 \mathrm{HC}$ participants were included in overall SZ-HC comparisons. A sub-cohort of $61 \mathrm{SZ}$ participants were included to test our primary hypothesis in subsequent analyses focused on functional impairments. Our SZ cohort demonstrated significantly lower scores across neurocognitive and social cognitive assessments, consistent with prior studies with the COBRE dataset (Avram et al., 2018; Cabral et al., 2016; Wang et al., 2015). We observed three principal components ( $\mathrm{PC} 1, \mathrm{PC} 2, \mathrm{PC} 3)$ that explained a significant portion of the variance across clinical and cognitive measures (Table 1). Additional demographic and clinical data on our low and high functioning subgroups defined by each PC are displayed in Supplementary Table 1. We observed no significant difference in age, sex, antipsychotic medication, or use of antidepressant medications ( $p>0.05)$ between low- and highfunctioning groups for each of our PCs of interest. Each PC represented a unique contribution to functional impairment in $\mathrm{SZ}$ and was examined in relation to neuroimaging measures summarized below in independent analyses. In accordance with the binary classification of functioning in our previous work (Tarcijonas et al., 2019), lowand highfunctioning SZ participants were defined based on loadings onto each of these components, which facilitated comparisons with the $\mathrm{HC}$ group. Consistent with previous studies, our SZ cohort exhibited greater FD, relative to the HC cohort (Table $1 ; \mathrm{p}<0.001$ ).

\subsection{Corticopallidal Connectivity: Independent Confirmation}

We first examined whether there are differences between $\mathrm{SZ}$ and $\mathrm{HC}$ groups in functional connectivity between GP ROIs and combined nodes within the salience network. The SZ cohort demonstrated significantly less connectivity between both left and right GP externa ROIs with nodes representing the salience network ( $\mathrm{P}<0.05$, Bonferroni corrected). Our primary analysis tested the hypothesis that functional connectivity between the GP and nodes of the salience network that emerged in our previous analyses will also differentiate low-functioning individuals with SZ from HC participants in the COBRE dataset. Connectivity between the GP and salience network ROIs was extracted for SZ and HC participants, and subsequently, examined between low-functioning, high-functioning participants and $\mathrm{HC}$ groups (Figure 1). For PC1, we observed decreased connectivity between left and right GP externa and the salience network in low-functioning SZ relative HC participants $(\mathrm{P}<0.05$, Bonferroni corrected; Figure 2). PC2 showed a similar relationship of decreased connectivity between the right GP externa and the salience between in the low-functioning SZ group relative to HC participants, but also a decrease in left GP externa-salience network connectivity between high-functioning SZ and $\mathrm{HC}$ participants ( $\mathrm{p}<0.05$, Bonferroni corrected). For PC3, we also observed decreased functional connectivity relative to $\mathrm{HC}$ participants in the low-functioning SZ group for both right and left GP externa-salience network connectivity ( $\mathrm{P}<0.05$, Bonferroni corrected). We additionally observed decreased connectivity between high-functioning SZ participants and the HC group in right GP externa-salience network connectivity. These results replicate our previous findings (Tarcijonas et al., 2019). However, significant results related to functional impairment were only demonstrated with our GP externa ROIs, contrary to our previous 
study, in which the interna ROIs were most prominent. Of note, no significant results were noted between low- and high-functioning individuals with SZ. In post-hoc analysis, none of the findings described above were significantly related to FD, antipsychotic medication exposure, age, or illness duration $(\mathrm{P}>0.1)$.

\subsection{Exploratory Group Analyses}

In addition to testing our previous corticopallidal connections in relation to functional impairment, we also examined whole-brain, voxel-wise connections of the GP. Results are summarized in Supplementary Tables 2-4 and displayed in Figure 3. For PC1 we observed decreased GP-salience network connectivity in low-functioning SZ participants in comparison with the $\mathrm{HC}$ group, robustly circumscribed in canonical regions of a salience network, and the medial dorsal nucleus of the thalamus, bilaterally (Figure 3). Lowfunctioning SZ cohorts defined by PC2 and PC3 also showed decreased GP connectivity with regions of the cingulate and supramarginal gyrus, relative to HCs.

\section{DISCUSSION}

In this study, our goal was to confirm prior results linking corticopallidal connectivity with functional impairment in a cohort of participants with chronic SZ. Proxy measures that represented functional impairment were derived to leverage available data from a publicly available dataset of participants with chronic SZ that lacks a direct assessment of functioning. In our primary analyses, we hypothesized, based on our previous findings, that GP-salience network connectivity would be abnormal in participants with lower scores of functioning relative to a matched control group. Confirming these previous results, we observed decreased functional connectivity between the GP and the salience network in SZ participants with lower loadings onto factors that represent measures that contribute to functional impairment in SZ. In additional exploratory analyses, we examined whether corticopallidal interactions are present outside of our a priori connections at a voxel-wise level. Not only did we observe regions circumscribed to the salience network, but found additional cortical regions associated with low functioning that largely mirrored group differences from our previous work, underscoring known CBGTC circuitry. Of note, we observed a consistent corticopallidal signal across all three unique PCs derived from measures that contribute to functional impairments in SZ. Our work is significant for its novel approach toward examining functional impairment with an external and publicly available dataset and its confirmation of our prior work (Tarcijonas et al., 2019). Replication studies, such as this study are crucial for biomarker development, especially with use of independent sources of data, and heterogenous measures of clinical phenomena.

Our finding of lower connectivity between the GP and salience network in lower-functioning patients may reflect an imbalance between the inhibitory indirect pathway and the excitatory direct pathways of the basal ganglia. This sort of imbalance may result in a less active direct pathway, affecting the ability to incite goal-directed behavior (Dunovan and Verstynen, 2016). In contrast to our prior work in first-episode SZ, one considerable difference in the present set of results is that significant relationships between overall functional impairment and corticopallidal connectivity were limited to the GP externa, not the interna. We speculate 
that this discrepancy may be due to inherent differences between these two cohorts, which capture distinct phases of illness. The COBRE dataset includes patients with chronic SZ, and overall, an extensive history of illness and medication exposure. The previous study included minimally treated individuals, including a sub-cohort of treatment naïve individuals, with first episode SZ. Differences in results may reflect dynamic effects of illness that change the topography of neural processing through the basal ganglia. As SZ illness progresses, there may be movement of neural circuitry from interna to exerna pathways. Future studies focused on white matter tracts through the globus pallidus may help address this question.

Our results contribute to our understanding of the role of the CBGTC system in clinical outcomes. While global connectivity studies remain inconclusive and may be intact, focusing on functional connectivity on substructures and specific systems is still relevant for biomarker development in SZ research. Functional connectivity of the striatum has been shown to be related to antipsychotic treatment response (Hadley et al., 2014; Sarpal et al., 2016; Sarpal et al., 2015). Thalamic connectivity has shown promise as a trait-based marker of SZ illness (Giraldo-Chica and Woodward, 2017). However, here, we focus on the pallidum - a relatively unexplored structure in SZ research that is a treatment target for a variety of neurologic conditions. The GP is a critical component of the CBGTC system with a distinct role in generating action from competing striatal inputs. Aberrant GP functioning in lesion studies is linked to deficits in motivational salience and goal-directed behavior, which are core components of the negative and cognitive symptoms of schizophrenia and contribute to functional deficits of the disorder (Arimura et al., 2013; Justin Rossi et al., 2017; Miller et al., 2006; Piron et al., 2016). In addition, deep brain stimulation of pallidal substructures may hold potential as a therapeutic strategy for individuals with schizophrenia who demonstrate more extreme functional limitations (Gault et al., 2018). Our results show a notable resemblance to findings in patients with akinetic mutism, who display poor functioning and phenotypic elements similar to the negative symptoms observed in schizophrenia (Darby et al., 2018). While more work is needed to further characterize the link between the GP and the salience network, the present findings suggest that it may relate play a role in neurocognitive domains such as motivation and goal-oriented behavior. We also noted findings within the higher-order associated regions of the supramarginal gyrus at the temporoparietal junction, consistent with our previous work, indicating possible deficits in low-functioning individuals with SZ in more extended regions of the attention system, language processing, and social cognition (Adolphs, 2001; Binder et al., 1997; Corbetta et al., 2008; Corbetta and Shulman, 2002; Tomasi and Volkow, 2012). Of note, the observed relationship between low-functioning patients and reduced GP-salience network connectivity was not significantly associated with symptom severity at baseline, suggesting that this relationship is independent of acute psychosis.

There are several limitations to the present work. For one, our measures of functioning were proxy measures derived from factors known to contribute to impairment in individuals with SZ. Reliability of this approach will need to be established in future studies. Thus, our findings are not a true replication given the proxy measure for functional impairments. An externally validated measure of functional outcomes consistent with our previous work would be ideal (Strauss and Carpenter, 1972), along with other community-based measures of outcome (Hogarty et al., 1974; Schooler N et al., 1979). Secondly, our study examined a 
cross-sectional cohort of patients, stable and chronically ill, with a long duration of treatment. Future longitudinal samples that combine neuroimaging with clinically rigorous measures of outcomes, as in our previous analysis are needed. Despite these limitations, we are confident that our data reduction across cognitive and clinical measures reflect realworld functioning, and our neuroimaging results are well-founded given that they are hypothesis driven, and localized. These results do advance the potential for corticopallidal circuitry as a target for therapeutic development.

In summary, we show independent evidence, from a cohort of individuals with chronic SZ, that further supports a role for corticopallidal interactions in the functional impairments of schizophrenia. Further studies in both early and chronic patients are needed to disentangle discrepancies in GP connectivity between our two studies. Nonetheless, corticopallidal connectivity may be a treatment target for detrimental functional outcomes often observed in chronic psychotic disorders, possibly via neuromodulatory methods. Future work focused on mechanistic clinical trials is necessary.

\section{Supplementary Material}

Refer to Web version on PubMed Central for supplementary material.

\section{Acknowledgement}

The authors would like to acknowledge all members of the Psychosis Recruitment and Assessment Core at the University of Pittsburgh who provide ongoing research assistance.

Funding source

This project was supported by the Brain \& Behavior Research Foundation (D.K.S.) and the National Institutes of Health through grant K23MH110661(D.K.S.). The publicly available imaging data and phenotypic information used for these analyses were collected and shared by the Mind Research Network and the University of New Mexico, funded by a National Institute of Health COBRE grant: P20RR021938.

\section{REFERENCES}

Adolphs R, 2001 The neurobiology of social cognition. Curr Opin Neurobiol 11(2), 231-239. [PubMed: 11301245]

Arimura N, Nakayama Y, Yamagata T, Tanji J, Hoshi E, 2013 Involvement of the globus pallidus in behavioral goal determination and action specification. J Neurosci 33(34), 13639-13653. [PubMed: 23966686]

Avram M, Brandl F, Bauml J, Sorg C, 2018 Cortico-thalamic hypo- and hyperconnectivity extend consistently to basal ganglia in schizophrenia. Neuropsychopharmacology.

Binder JR, Frost JA, Hammeke TA, Cox RW, Rao SM, Prieto T, 1997 Human brain language areas identified by functional magnetic resonance imaging. J Neurosci 17(1), 353-362. [PubMed: 8987760]

Bowie CR, Reichenberg A, Patterson TL, Heaton RK, Harvey PD, 2006 Determinants of real-world functional performance in schizophrenia subjects: correlations with cognition, functional capacity, and symptoms. Am J Psychiatry 163(3), 418-425. [PubMed: 16513862]

Cabral C, Kambeitz-Ilankovic L, Kambeitz J, Calhoun VD, Dwyer DB, von Saldern S, Urquijo MF, Falkai P, Koutsouleris N, 2016 Classifying Schizophrenia Using Multimodal Multivariate Pattern Recognition Analysis: Evaluating the Impact of Individual Clinical Profiles on the Neurodiagnostic Performance. Schizophr Bull 42 Suppl 1, S110-117. [PubMed: 27460614] 
Calhoun VD, Sui J, Kiehl K, Turner J, Allen E, Pearlson G, 2011 Exploring the psychosis functional connectome: aberrant intrinsic networks in schizophrenia and bipolar disorder. Front Psychiatry 2, 75. [PubMed: 22291663]

Corbetta M, Patel G, Shulman GL, 2008 The reorienting system of the human brain: from environment to theory of mind. Neuron 58(3), 306-324. [PubMed: 18466742]

Corbetta M, Shulman GL, 2002 Control of goal-directed and stimulus-driven attention in the brain. Nat Rev Neurosci 3(3), 201-215. [PubMed: 11994752]

Cox RW, 1996 AFNI: software for analysis and visualization of functional magnetic resonance neuroimages. Comput Biomed Res 29(3), 162-173. [PubMed: 8812068]

Da Cunha C, Boschen SL, Gomez AA, Ross EK, Gibson WS, Min HK, Lee KH, Blaha CD, 2015 Toward sophisticated basal ganglia neuromodulation: Review on basal ganglia deep brain stimulation. Neurosci Biobehav Rev 58, 186-210. [PubMed: 25684727]

Darby RR, Joutsa J, Burke MJ, Fox MD, 2018 Lesion network localization of free will. Proc Natl Acad Sci U S A.

Dunovan K, Verstynen T, 2016 Believer-Skeptic Meets Actor-Critic: Rethinking the Role of Basal Ganglia Pathways during Decision-Making and Reinforcement Learning. Front Neurosci 10, 106. [PubMed: 27047328]

Fett AK, Viechtbauer W, Dominguez MD, Penn DL, van Os J, Krabbendam L, 2011 The relationship between neurocognition and social cognition with functional outcomes in schizophrenia: a metaanalysis. Neurosci Biobehav Rev 35(3), 573-588. [PubMed: 20620163]

Gault JM, Davis R, Cascella NG, Saks ER, Corripio-Collado I, Anderson WS, Olincy A, Thompson JA, Pomarol-Clotet E, Sawa A, Daskalakis ZJ, Lipsman N, Abosch A, 2018 Approaches to neuromodulation for schizophrenia. J Neurol Neurosurg Psychiatry 89(7), 777-787. [PubMed: 29242310]

Giraldo-Chica M, Woodward ND, 2017 Review of thalamocortical resting-state fMRI studies in schizophrenia. Schizophr Res 180, 58-63. [PubMed: 27531067]

Global Burden of Disease Study, C., 2015 Global, regional, and national incidence, prevalence, and years lived with disability for 301 acute and chronic diseases and injuries in 188 countries, 19902013: a systematic analysis for the Global Burden of Disease Study 2013. Lancet 386(9995), $743-$ 800. [PubMed: 26063472]

Gorgolewski K, Burns CD, Madison C, Clark D, Halchenko YO, Waskom ML, Ghosh SS, 2011 Nipype: a flexible, lightweight and extensible neuroimaging data processing framework in python. Front Neuroinform 5, 13. [PubMed: 21897815]

Green MF, 1996 What are the functional consequences of neurocognitive deficits in schizophrenia? Am J Psychiatry 153(3), 321-330. [PubMed: 8610818]

Guy W, Bonato R, CGI: Clinical Global Impressions., in: US Department of Health, E.a.W., ADAMHA, NIMH Psychopharmacology Research Branch (Ed.), Rockville, MD, pp. 217-222.

Hadley JA, Nenert R, Kraguljac NV, Bolding MS, White DM, Skidmore FM, Visscher KM, Lahti AC, 2014 Ventral tegmental area/midbrain functional connectivity and response to antipsychotic medication in schizophrenia. Neuropsychopharmacology 39(4), 1020-1030. [PubMed: 24165885]

Hallquist MN, Hwang K, Luna B, 2013 The nuisance of nuisance regression: spectral misspecification in a common approach to resting-state fMRI preprocessing reintroduces noise and obscures functional connectivity. Neuroimage 82, 208-225. [PubMed: 23747457]

Hogarty GE, Goldberg SC, Schooler NR, 1974 Drug and sociotherapy in the aftercare of schizophrenic patients. III. Adjustment of nonrelapsed patients. Arch Gen Psychiatry 31(5), 609-618. [PubMed: 4374156]

Jaaskelainen E, Juola P, Hirvonen N, McGrath JJ, Saha S, Isohanni M, Veijola J, Miettunen J, 2013 A systematic review and meta-analysis of recovery in schizophrenia. Schizophr Bull 39(6), 12961306. [PubMed: 23172003]

Jenkinson M, Beckmann CF, Behrens TE, Woolrich MW, Smith SM, 2012 Fsl. Neuroimage 62(2), 782-790. [PubMed: 21979382]

Justin Rossi P, Peden C, Castellanos O, Foote KD, Gunduz A, Okun MS, 2017 The human subthalamic nucleus and globus pallidus internus differentially encode reward during action control. Hum Brain Mapp 38(4), 1952-1964. [PubMed: 28130916] 
Kay SR, Fiszbein A, Opler LA, 1987 The positive and negative syndrome scale (PANSS) for schizophrenia. Schizophr Bull 13(2), 261-276. [PubMed: 3616518]

Mayer JD, Salovey P, Caruso DR, Sitarenios G, 2003 Measuring emotional intelligence with the MSCEIT V2.0. Emotion 3(1), 97-105. [PubMed: 12899321]

Meyer-Lindenberg A, Miletich RS, Kohn PD, Esposito G, Carson RE, Quarantelli M, Weinberger DR, Berman KF, 2002 Reduced prefrontal activity predicts exaggerated striatal dopaminergic function in schizophrenia. Nat Neurosci 5(3), 267-271. [PubMed: 11865311]

Miller JM, Vorel SR, Tranguch AJ, Kenny ET, Mazzoni P, van Gorp WG, Kleber HD, 2006 Anhedonia after a selective bilateral lesion of the globus pallidus. Am J Psychiatry 163(5), 786-788. [PubMed: 16648316]

Nuechterlein KH, Green MF, Kern RS, Baade LE, Barch DM, Cohen JD, Essock S, Fenton WS, Frese FJ 3rd, Gold JM, Goldberg T, Heaton RK, Keefe RS, Kraemer H, Mesholam-Gately R, Seidman LJ, Stover E, Weinberger DR, Young AS, Zalcman S, Marder SR, 2008 The MATRICS Consensus Cognitive Battery, part 1: test selection, reliability, and validity. Am J Psychiatry 165(2), 203-213. [PubMed: 18172019]

Parvizi J, Rangarajan V, Shirer WR, Desai N, Greicius MD, 2013 The will to persevere induced by electrical stimulation of the human cingulate gyrus. Neuron 80(6), 1359-1367. [PubMed: 24316296]

Patel AX, Kundu P, Rubinov M, Jones PS, Vertes PE, Ersche KD, Suckling J, Bullmore ET, 2014 A wavelet method for modeling and despiking motion artifacts from resting-state fMRI time series. Neuroimage 95, 287-304. [PubMed: 24657353]

Piron C, Kase D, Topalidou M, Goillandeau M, Orignac H, N'Guyen TH, Rougier N, Boraud T, 2016 The globus pallidus pars interna in goal-oriented and routine behaviors: Resolving a long-standing paradox. Mov Disord 31(8), 1146-1154. [PubMed: 26900137]

Reddy LF, Horan WP, Green MF, 2016 Motivational Deficits and Negative Symptoms in Schizophrenia: Concepts and Assessments. Curr Top Behav Neurosci 27, 357-373. [PubMed: 26164592]

Robinson DG, Woerner MG, McMeniman M, Mendelowitz A, Bilder RM, 2004 Symptomatic and functional recovery from a first episode of schizophrenia or schizoaffective disorder. Am J Psychiatry 161(3), 473-479. [PubMed: 14992973]

Sarpal DK, Argyelan M, Robinson DG, Szeszko PR, Karlsgodt KH, John M, Weissman N, Gallego JA, Kane JM, Lencz T, Malhotra AK, 2016 Baseline Striatal Functional Connectivity as a Predictor of Response to Antipsychotic Drug Treatment. Am J Psychiatry 173(1), 69-77. [PubMed: 26315980]

Sarpal DK, Robinson DG, Lencz T, Argyelan M, Ikuta T, Karlsgodt K, Gallego JA, Kane JM, Szeszko PR, Malhotra AK, 2015 Antipsychotic treatment and functional connectivity of the striatum in first-episode schizophrenia. JAMA Psychiatry 72(1), 5-13. [PubMed: 25372846]

Schooler N, Weissman M,G,H., 1979 Social adjustment scale for schizophrenics . National Institute of Mental Health, Rockville, MD.

Strauss JS, Carpenter WT Jr., 1972 The prediction of outcome in schizophrenia. I. Characteristics of outcome. Arch Gen Psychiatry 27(6), 739-746. [PubMed: 4637891]

Tarcijonas G, Foran W, Haas GL, Luna B, Sarpal DK, 2019 Intrinsic Connectivity of the Globus Pallidus: An Uncharted Marker of Functional Prognosis in People With First-Episode Schizophrenia. Schizophr Bull.

Tomasi D, Volkow ND, 2012 Resting functional connectivity of language networks: characterization and reproducibility. Mol Psychiatry 17(8), 841-854. [PubMed: 22212597]

Wang HL, Rau CL, Li YM, Chen YP, Yu R, 2015 Disrupted thalamic resting-state functional networks in schizophrenia. Front Behav Neurosci 9, 45. [PubMed: 25762911]

Wechsler D, 1999 Wechsler Abbreviated Scale of Intelligence The Psychological Corporation: Harcourt Brace \& Company, New York, NY.

Wechsler D, 2008 Wechsler Adult Intelligence Scale-Fourth Edition The Psychological Corporation., San Antonio, TX.

Wojtalik JA, Smith MJ, Keshavan MS, Eack SM, 2017 A Systematic and Meta-analytic Review of Neural Correlates of Functional Outcome in Schizophrenia. Schizophr Bull 43(6), 1329-1347. [PubMed: 28204755] 
Yarkoni T, Poldrack RA, Nichols TE, Van Essen DC, Wager TD, 2011 Large-scale automated synthesis of human functional neuroimaging data. Nat Methods 8(8), 665-670. [PubMed: 21706013] 
A)

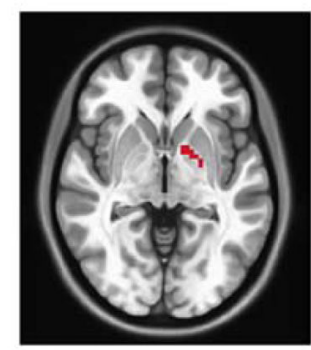

Left Externa

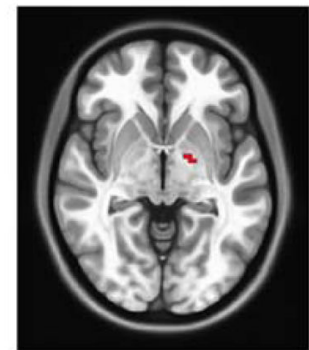

Left Interna

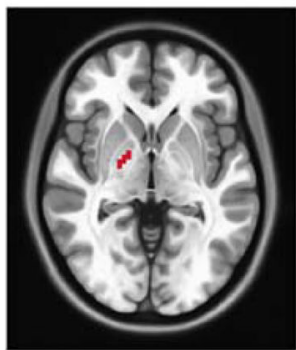

Right Interna

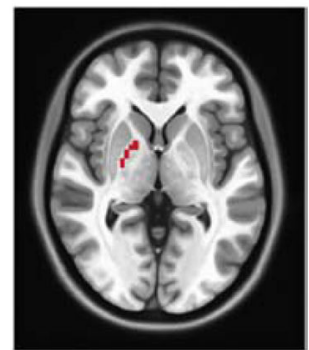

Right Externa

B)

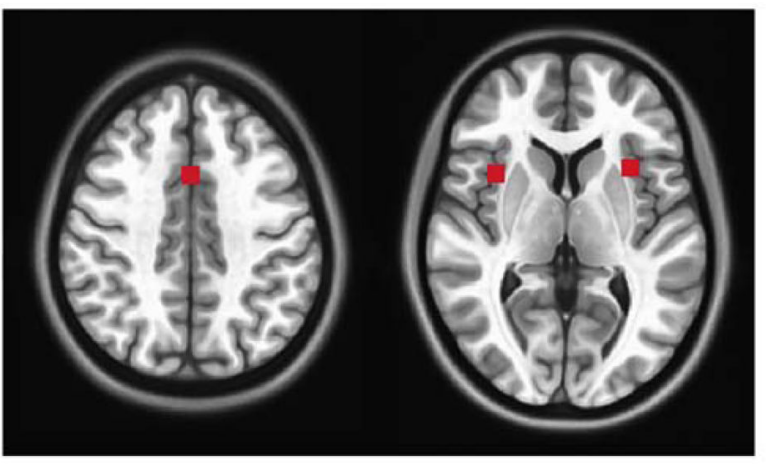

$z=42$

$z=5$

Figure 1. Regions of Interest

In A) we display GP seed regions used for connectivity analyses. In B) nodes within the salience network derived from our previous findings are depicted. These nodes were combined for our GP connectivity analyses. 
Left GP externa

Component 1
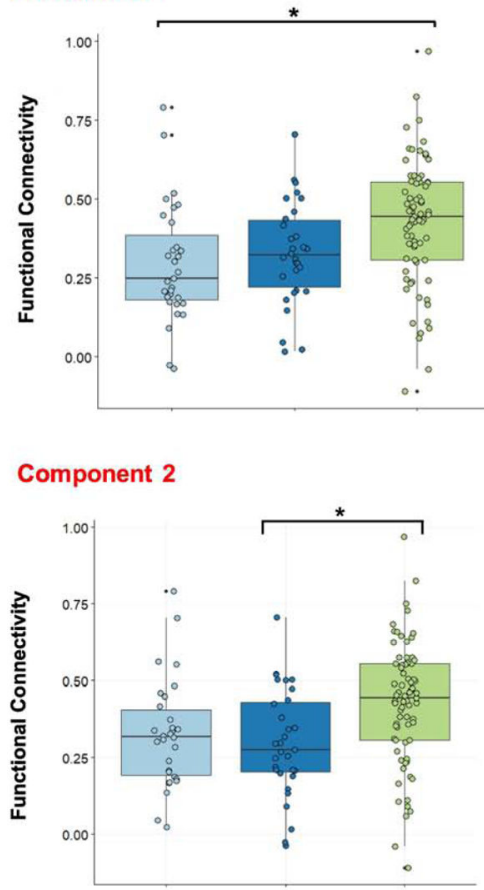

Component 3

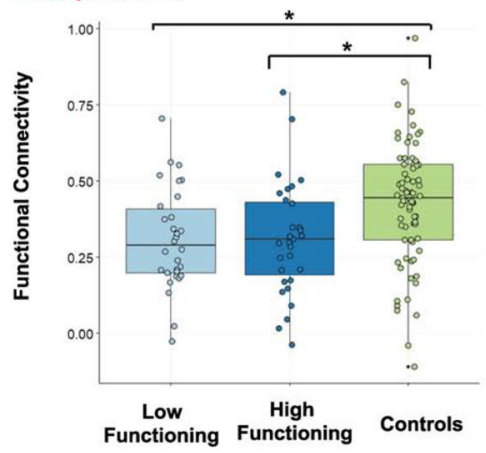

Right GP externa
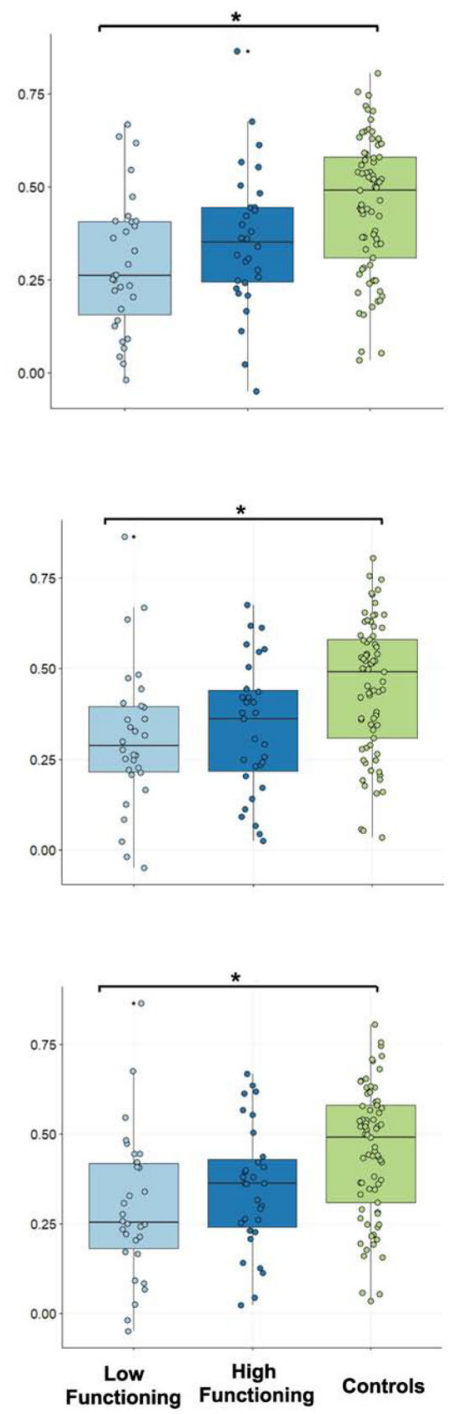

Figure 2. Corticopallidal connectivity results.

Results of our primary analyses are displayed. We observed a significant decrease in GPsalience network functional connectivity between healthy control and lower functioning SZ participants. This result was consistent across all groupings of low and high-functioning defined by each of our 3 PCs of interest. 


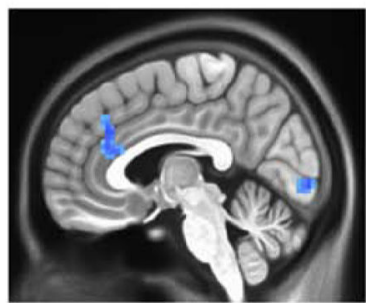

Left GP interna

$X=4$

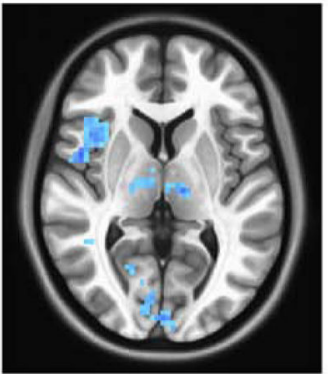

Left GP externa

$z=5$

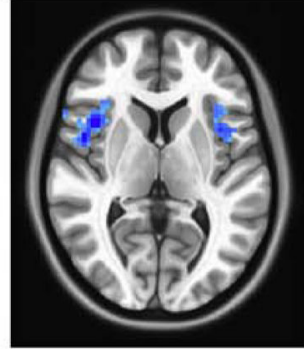

Right GP externa

$z=6$

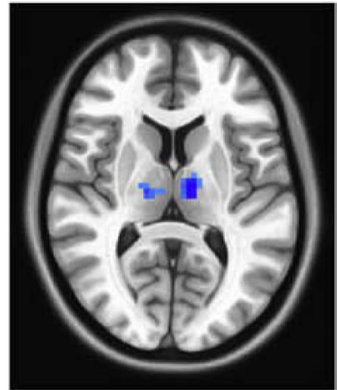

Right GP interna

Figure 3. Exploratory voxel-wise analyses.

Images illustrating key results from voxel-wise analyses are displayed. 
Table 1.

PCA details: weights of relevant components.

\begin{tabular}{|l|l|l|l|}
\hline & Component 1 & Component 2 & Component 3 \\
\hline CGI & -0.17367 & -0.66417 & -0.16769 \\
\hline WASI & 0.525341 & -0.1718 & -0.02039 \\
\hline MSCEIT & 0.32916 & -0.19875 & 0.598223 \\
\hline WAIS & 0.46972 & -0.04151 & -0.27565 \\
\hline PANSS Positive & -0.11662 & -0.57911 & -0.37896 \\
\hline PANSS Negative & -0.20475 & -0.36209 & 0.627686 \\
\hline MCCB & 0.556191 & -0.14716 & -0.00272 \\
\hline
\end{tabular}

CGI: Clinical Global Impression; WASI: Wechsler Abbreviated Scale of Intelligence; MSCEIT: Mayer-Salovey-Caruso Emotional Intelligence Test; WAIS: Wechsler Adult Intelligence Scale; PANSS: Positive and Negative Syndrome Scale; MCCB: Measurement and Treatment Research to Improve Cognition in Schizophrenia Consensus Cognitive Battery. 
Table 2.

Demographic and clinical characteristics of participants

\begin{tabular}{|c|c|c|c|c|c|c|c|c|}
\hline & \multicolumn{3}{|c|}{ Patients $(\mathrm{N}=72)$} & \multicolumn{3}{|c|}{ Controls $(N=73)$} & \multirow[b]{2}{*}{$\mathrm{T} / X^{2}$} & \multirow[b]{2}{*}{ p-value } \\
\hline & Mean & SD & $\mathbf{N}$ & Mean & SD & $\mathbf{N}$ & & \\
\hline Age (years) & 38.17 & 13.89 & & 35.89 & 11.65 & & -1.07 & 0.29 \\
\hline Sex & & & & & & & 2.78 & 0.10 \\
\hline Male & & & 58 & & & 50 & & \\
\hline Female & & & 14 & & & 23 & & \\
\hline WASI IQ & 100.30 & 16.47 & & 110.98 & 13.33 & & 4.09 & $<0.001$ \\
\hline WAIS PSI & 87.91 & 13.32 & & 101.84 & 14.55 & & 5.68 & $<0.001$ \\
\hline MCCB & 33.17 & 14.11 & & 49.05 & 8.92 & & 7.46 & $<0.001$ \\
\hline MSCEIT & 41.58 & 12.48 & & 51.26 & 9.76 & & 4.96 & $<0.001$ \\
\hline CGI & 3.57 & 0.87 & & NA & NA & & NA & $\mathrm{NA}$ \\
\hline Negative symptoms (PANSS) & 14.53 & 4.83 & & NA & NA & & NA & $\mathrm{NA}$ \\
\hline Positive symptoms (PANSS) & 14.96 & 4.83 & & NA & NA & & NA & NA \\
\hline Framewise Displacement & 0.412 & 0.30 & & 0.28 & 0.15 & & -3.43 & $<0.001$ \\
\hline chlorpromazine equivalents (mg) & 368.24 & 304.25 & & & & & & NA \\
\hline
\end{tabular}

CGI: Clinical Global Impression; WASI: Wechsler Abbreviated Scale of Intelligence; MSCEIT: Mayer-Salovey-Caruso Emotional Intelligence Test; WAIS: Wechsler Adult Intelligence Scale; PANSS: Positive and Negative Syndrome Scale; MCCB: Measurement and Treatment Research to Improve Cognition in Schizophrenia Consensus Cognitive Battery. 